\title{
Inquietud y pensamiento
}

\author{
Uneasiness and Thought
}

\author{
FACUNDO CASUllo \\ Universidad de Buenos Aires/UNLP, Argentina
}

RESUMEN. Este trabajo busca caracterizar los vínculos que en la historia del pensamiento moderno han acompañado la noción, cada vez más relevante, de inquietud. Para esto, veremos cómo funciona la inquietud en tres autores modernos muy distintos entre sí como son Locke, Hegel (leído a través Hyppolite) y Foucault. Este recorrido nos permitirá ubicar la inquietud en el cruce de las relaciones entre pensamiento, deseo, poder y la libertad.

Palabras clave: Inquietud, Locke, Hegel, Hyppolite, Foucault, pensamiento, deseo, ascesis.

\section{Introducción}

En el número 41 de esta revista, D. Vernazza publicó un artículo sobre la inquietud en Leibniz y Montesquieu. La filosofía contemporánea da varios ejemplos de coincidencias que no pueden ser pensadas en términos de influencia ${ }^{1}$. Se ha sugerido para esto la noción de confluencia, mejor para dar cuenta del cruce «casual» entre diversas líneas de pensamiento. Sin embargo, aunque algo del orden de la confluencia pueda considerarse, no podemos ignorar el hecho de que la noción de inquietud se está presentando en diversos campos del pensamiento francés contemporáneo. En la Antropología, D. Fassin enmarca uno de sus estudios ${ }^{2}$ en una
ABSTRACT. This paper aims to characterize the links that have accompanied in the history of modern though a notion that appears to have a growing relevancy in the present: the notion of «uneasiness». In order to do this, we will examine how the uneasiness functions in three modern thinkers, very different amongst them such as Locke, Hegel (through Hyppolite's work) and Foucault. This path will allow us to locate the uneasiness in the space opened by the relationships between thought, desire, power and freedom.

Key words: Uneasiness, Locke, Hegel, Hyppolite, Foucault, thought, desire, asceticism.

oposición inquietud-ansiedad, que enfrenta la primera noción a un diagnóstico de la modernidad realizado por W. $\mathrm{H}$. Auden en términos de ansiedad ${ }^{3}$. En teoría política y social, el artículo de Vernazza marca un sendero que deberá sin duda continuarse, pero que tiene al menos un antecedente relevante en la teoría social. En efecto, el libro de E. Castelli Gattinara, Les inquiétudes de la raison ${ }^{4}$ señala la relevancia de la inquietud para la comprensión de la contemporaneidad en ámbitos como la historia y la epistemología. La filosofía no está exenta de este avance: J.-L. Nancy y M. Potte-Bonneville tratan el tema en dos autores que aquí recuperamos desde otra perspectiva ${ }^{5}$. En función de esto, lo mínimo que puede de- 
cirse es que la inquietud aparece cada vez más como una noción capaz de alumbrar el pensamiento francés contemporáneo, sin duda en detrimento de otras, como la ansiedad, la angustia o la contradicción, que ya no parecen tener la fuerza que alguna vez las caracterizó.

En lo que sigue nos proponemos mostrar las relaciones que han caracterizado a esta noción de inquietud en la historia del pensamiento moderno a través de tres autores muy diferentes entre sí. En primer lugar, veremos cómo surge en Locke una idea de inquietud que tendrá un eco importante en el pensamiento alemán y que luego llegará a Francia. En segundo lugar, veremos a través de la lectura que Hyppolite realiza de Hegel, cómo funciona la inquietud en un pensamiento que ya no concibe la subjetividad en términos cartesianos sino que la sitúa en un conocimiento del absoluto. Finalmente, la figura de Hyppolite nos permitirá el pasaje a un pensamiento de la inquietud en el que ni la totalidad ni la subjetividad tienen un papel relevante, como es el caso de Foucault.

\section{Locke: «El deseo es inquietud»}

\section{Poder, Libertad y Pensamiento}

En Ensayo concerniente al entendimiento humano vemos emerger la inquietud como una noción central en el espacio abierto por los vínculos entre poder, necesidad y libertad ${ }^{6}$. Locke explica allí cómo tienen lugar la necesidad, propio del movimiento de lo sensible, y la libertad, capaz de caracterizar el movimiento del pensamiento. La idea del poder es adquirida por la mente gracias a la percepción que ésta tiene del devenir de sus propias ideas y de las cosas. Observando por una parte cómo algunas cosas comienzan a existir y otras sufren todo tipo de alteraciones o cesan de existir, y, por otra parte, el hecho de que mucho de estos cambios tienen lugar gracias a la propia elección, la mente considera la posibilidad de efectuar cambios en el devenir. El poder es aquí una relación; y más específicamente, relación con el cambio. Es a partir del cambio y del movimiento que la reflexión sobre el poder tendrá lugar. De allí que la mente conciba un poder con dos caras: una pasiva, en relación al movimiento sensible y a la percepción, y otra activa, que da cuenta del poder que encontramos en nosotros mismos de «comenzar o impedir, continuar o terminar varias acciones de nuestras mentes, y movimientos de nuestros cuerpos» ${ }^{7}$. La facultad de la mente a considerar en este punto será, por supuesto, la voluntad (Will), puesto que si una acción se realiza sin que haya una elección de la mente esta acción resulta involuntaria.

Ahora bien, este poder de comenzar, continuar o terminar diversas acciones en nuestra mente o movimientos de nuestro cuerpo por propia elección plantea el problema de la libertad y de la necesidad. Así, sostiene Locke «mientras el hombre tiene el poder de pensar, o no pensar; mover, o no mover, de acuerdo a la preferencia o dirección de su propia mente; el hombre es libre» ${ }^{8}$. La libertad consiste así en la posesión de un poder efectivo para realizar la voluntad. Un hombre que cae de una torre puede sin dudas tener la voluntad de no caer, sin embargo, «no estando en su poder el impedimento de este movimiento» ${ }^{9}$, este hombre no es libre de dejar de caer. Por este motivo, Locke sostiene la «libertad no puede estar donde no hay pensamiento, ni volición, ni voluntad; pero puede haber pensamiento, puede haber voluntad, puede haber volición, donde no hay libertad» ${ }^{10}$. El hombre que cae de una torre está, con respecto a su caída, en un estado de necesidad, ya que carece del poder de modificar dicho movimiento a partir del pensamiento. Se establece así un vínculo estrecho entre 
libertad, pensamiento y cambio. Sólo hay libertad en la medida en que se tiene el poder de efectuar un cambio a partir del pensamiento. Puede haber pensamiento sin libertad, así como puede incluso haber voluntad sin libertad: la libertad implica un poder extra, que no se da necesariamente con el pensamiento y la voluntad (aunque no pueda darse sin ellos).

\section{Inquietud y Deseo}

En este marco, la pregunta por la libertad de la voluntad no tiene sentido. Tanto la libertad como la voluntad son poderes, y los poderes pertenecen a agentes, no a otros poderes. Podemos preguntarnos si un hombre es libre o no lo es, pero no si la voluntad de un hombre es libre. El hombre, como agente, es libre si tiene el poder de hacer efectiva su voluntad ${ }^{11}$. Sin embargo, el hombre no es libre con respecto a su voluntad, es decir que no es libre de preferir la existencia o inexistencia de una acción (aunque no pueda realizarla) que su voluntad manda. Nos encontramos en este punto con una distinción central para nuestro análisis. Con respecto a la acción de la voluntad, el hombre está bajo necesidad. Lo que nos plantea la siguiente pregunta: ¿Qué es lo que determina la voluntad? ${ }^{12}$ Si la libertad consiste en poder hacer efectivo lo que la voluntad manda, el problema ontológico de la determinación de la voluntad deviene un problema ético de la mayor importancia. La respuesta de Locke es clara: «Y a esto respondo, el motivo para continuar en el mismo estado o acción, es sólo la satisfacción presente en él; el motivo para cambiar, es siempre alguna inquietud» ${ }^{13}$.

Aunque esta noción de uneasiness ha sido traducida al español como males$\operatorname{tar}^{14}$, veremos que corresponde a lo que Hegel llamará Unruhe y que Jean Hyppolite volverá a volcar al francés como inquiétude ${ }^{15}$. Adelantándonos un poco po- demos decir que es también esta misma inquietud que Foucault transformará en el punto clave de su relación con el deseo. Más allá de las traducciones esta noción se halla siempre ligada a un campo semántico que refiere a una falta de quietud, de reposo, de comodidad o de tranquilidad por causas que pueden ir desde una mínima preocupación, agitación, inestabilidad, o incertidumbre hasta llegar a lo espantoso y a lo tormentoso. La inquietud comporta diversos grados, de modo que tiene un valor ambiguo: positivo en la medida en que nos induce a la acción, negativo en la medida que es aquello que toda acción pretende anular al instaurar un estado de satisfacción.

Esta relación, o mejor dicho no-relación entre satisfacción e inquietud (puesto que nunca coexisten, nunca pueden darse simultáneamente), lleva a Locke a sostener: «tanto como haya deseo, tanto hay de inquietud» ${ }^{16}$. La inquietud implica siempre un deseo (de escapar a un estado o de alcanzar otro); más aún, si existe algún deseo en el hombre es porque tiene lugar en él cierta inquietud ${ }^{17}$. Del mismo modo, la inquietud es deseo; la inquietud causada por la ausencia de un bien o la presencia de un mal siempre es al mismo tiempo el deseo de alcanzar el bien ausente o de librarse del mal presente. Sin embargo, no todo bien propuesto al hombre implica necesariamente el deseo de alcanzarlo y por lo tanto tampoco implica necesariamente algún grado de inquietud. Así, la expectativa de una eterna vida de felicidad tras la muerte, bien con el cual ningún otro puede compararse, sólo mueve una pequeña cantidad de hombres a perseguirla. Para Locke, que la ausencia de un bien no implique la inquietud, como lo hace la presencia del mal, permite explicar al hombre que se dice: video meliora, proboque, deteriora sequor ${ }^{18}$. Sólo en tanto hay inquietud el hombre está determinado a actuar para modificar su estado. 


\section{Juicio y Libertad}

Hemos dicho que la libertad consiste en el poder de efectuar o no la voluntad según el pensamiento; pero, al mismo tiempo, hemos visto que no es posible atribuir libertad a la voluntad. En este sentido, la voluntad está sometida, a través de la inquietud, a la necesidad. Pero aparece ahora un aspecto distinto de la libertad. Durante el transcurso del capítulo encontramos que la oposición libertad-necesidad es remplazada por la oposición libertad-esclavitud. Un hombre que cae de una torre no es libre de modificar esa situación. Su estado se desarrollará bajo la más clara necesidad y su caída no se detendrá hasta el momento en que su cuerpo encuentre, dolorosamente, un apoyo. ¿Ocurre lo mismo con la inquietud? ¿Nuestros deseos nos someten a la misma necesidad que una caída? Se abre aquí la dimensión ética del asunto.

Existe un caso en el que un hombre puede ser libre con respecto a su voluntad: se trata de la suspensión de la acción en función de la consideración de las consecuencias en función de un bienestar futuro ${ }^{19}$. Puesto que el deseo es siempre deseo de alcanzar un bien ausente o liberarse de un mal presente, Locke puede argumentar que el deseo, finalmente, es deseo de felicidad: «Si se nos pregunta más aún, ¿qué mueve al deseo? Yo respondo, la felicidad, y sólo eso» ${ }^{20}$. Y puesto que la felicidad es definida como «el mayor placer del que somos capaces» ${ }^{21}$, y puesto que, como dijimos, no puede haber mayor bien futuro que la eterna felicidad prometida por Dios a los piadosos, tenemos ahora un criterio para la intervención del juicio (judgment). La posibilidad de la propia determinación a través del juicio permite el pasaje de la oposición libertad-necesidad a la oposición libertad-esclavitud: «cuanto más somos alejados de esta determinación [del juicio], tanto más cerca estamos de la miseria y la esclavitud» ${ }^{22}$.

El deseo es siempre movido por la felicidad, pero la felicidad no siempre es considerada detenida y adecuadamente. Por esto, el juicio aparece como aquello que permitirá determinar la verdadera felicidad. De este modo, Locke puede presentar una relación entre necesidad, felicidad verdadera y libertad: «La necesidad de perseguir la verdadera felicidad, el fundamento de toda libertad» ${ }^{23}$. El juicio nos impone la necesidad de la verdadera felicidad (no de la imaginaria, es decir, la que no ha sido debidamente considerada) como fundamento de la libertad.

El problema que surge entonces es el siguiente: ¿cómo ocurre que se tenga un juicio equivocado sobre el bien y el mal de una acción determinada? Si el nombre que damos a lo que nos produce placer no es otro que el de bien, y del mismo modo llamamos mal a aquello que nos produce dolor. ¿Cómo es posible que juzguemos mal en torno al placer y al dolor que experimentamos? La respuesta de Locke es interesante: el juicio sobre el bien y el mal se da en un doble sentido: por un lado, en torno al placer y al dolor presentes; por otro lado en torno al placer y al dolor que las consecuencias futuras de los deseos presentes puedan traer consigo ${ }^{24}$. Si consideramos los deseos desde el punto de vista de la vida eterna, resulta claro que la virtud debe ser preferida al vicio. De este modo: «Podemos cambiar el carácter agradable o desagradable en las cosas [...] Una correcta consideración alcanzará en algunos casos; y práctica, aplicación y costumbre en la mayoría» ${ }^{25}$.

Así, si bien no podemos atribuir libertad a nuestra voluntad, puesto que está determinada por la inquietud, resulta que el juicio abre la posibilidad de una suspensión para considerar las consecuencias de nuestros deseos. Sin embargo, el juicio aparece sólo como una primera 
instancia (suficiente en algunos casos). Pero la mayoría de las veces, el juicio deberá dar lugar a prácticas, aplicación y hábitos que permitirán la modificación de los deseos. De suerte que, para contestar la pregunta planteada al comienzo del parágrafo, nuestros deseos no nos someten a la misma necesidad de una caída, sino en todo caso a cierta esclavitud de la cual el hombre puede liberarse a través del juicio y la ascesis ${ }^{26}$. A través de un trabajo aplicado sobre nuestros hábitos y prácticas, el pensamiento podría llegar al dominio de los propios deseos.

Hegel a través de Hyppolite: «Esta inquietud es el sí mismo»

\section{Filosofía y límite}

La inquietud juega un rol importante en la filosofía de Hegel. Particularmente interesante resulta, desde esta perspectiva, la lectura de Jean Hyppolite. En efecto, no sólo su influencia en la recepción francesa de Hegel es fundamental ${ }^{27}$, sino que además Hyppolite puso de relieve esta noción de inquietud. $\mathrm{Su}$ aproximación a este problema nos permitirá mostrar diferencias y similitudes importantes con el planteo de Locke, y que a su vez nos permitirán llegar a un problema de la filosofía contemporánea: el del humanismo y el misticismo.

Por otra parte, en la medida en que nuestro recorrido apunta a la obra de Foucault, la figura de Hyppolite es inevitable. Profesor de Foucault en el khâgne ${ }^{28}$ (y director tanto de su primer trabajo universitario sobre Hegel, como de su tesis menor de doctorado sobre la antropología desde el punto de vista pragmático en Kant), su lectura antihumanista de Hegel tendrá una influencia fundamental sobre su alumno, quien al sucederlo en el Collège de France, inscribiría su propio trabajo en la línea de su profesor: «todos los problemas que son los nuestros [...] es él quien los estableció para nosotros» ${ }^{29}$. Foucault asume como problema principal de la obra de Hyppolite el de la finitud filosófica ${ }^{30}$. Y en efecto, la conciencia desgraciada, tema central según Hyppolite, es la expresión de toda conciencia que se encuentre ante el límite y la escisión: «La conciencia desgraciada es la subjetividad que aspira al descanso de la unidad» ${ }^{31}$. El Dios más allá, los otros hombres cuyos puntos de vista difieren del propio, la internalización de la escisión por parte de la autoconciencia, son los límites con los que la conciencia desgraciada tiene que lidiar en su búsqueda de la unidad. Pero en un sentido más general, la finitud filosófica significa la ineludible obligación por parte del filósofo a dar cuenta de la historicidad del pensamiento de lo universal. La conciencia de pensar la totalidad en y desde la historia, que la Fenomenología pretendía explicar, es así para Foucault la necesaria suspensión de dicha totalidad, y la clara imposibilidad de la objetividad.

\section{Inquietud, deseo y conciencia}

La inquietud es tratada por Hyppolite en el comentario de la parte B de la Fenomenología, «Autoconciencia». Las tres partes que componen este capítulo tienen como temas, respectivamente, la vida y el deseo, la lucha entre el amo y el esclavo, y la conciencia desgraciada, resultado esta última de la dialéctica completa de la autoconciencia. La autoconciencia es, ante todo, deseo: «La autoconciencia es deseo, pero lo que desea, sin saberlo todavía explícitamente, es ella misma [...] Tal es el deseo que se busca a sí mismo en lo otro, el deseo del reconocimiento del hombre por el hombre» ${ }^{32}$.

Ahora bien, el deseo (Begierde) tiene un sentido amplio. En primer lugar da cuenta del apetito, sensación que compartimos con los animales. Sin embargo, de lo que se trata aquí es de otra cosa: el 
deseo de sí mismo, el deseo del reconocimiento del otro, no puede definirse como simple apetito natural: «No hay que olvidar que este ser no es el ser de la naturaleza, es el ser del deseo, la inquietud del sí mismo, y que, por consiguiente, lo que debemos encontrar en el ser o actualizar en él es el modo de ser propio de la autoconciencia» ${ }^{33}$. Esta distinción entre el apetito (natural) y el deseo (espiritual) es fundamental para comprender la noción de inquietud:

«El ser de la vida no es la sustancia, sino más bien la inquietud del sí mismo. Según un reciente comentarista de la filosofía hegeliana, la intuición fundamental que dio origen a la filosofía hegeliana sería la movilidad de la vida. Nos parece que eso no es suficiente. El epíteto que más se repite en la dialéctica hegeliana es el de unruhig. La vida es inquietud, inquietud del sí mismo que se ha perdido y vuelve a encontrase en su alteridad [...] El ser del hombre "no es nunca lo que es, siempre es lo que no es"»" ${ }^{34}$.

Se trata de un deseo que caracteriza sólo al hombre, y que, en tanto tal, puede ser caracterizado como inquietud. La vida natural, aun con su constante movilidad, carece de inquietud. El devenir de culturas y pueblos no se explica suficientemente si tomamos la movilidad de la vida natural como modelo. Es necesario partir de la vida humana y de la inquietud que implica toda subjetividad en la medida en que experimenta la finitud, el límite que le impone ya sea un objeto cuya verdad quiere alcanzar, ya sea el resto de los hombres, cuyo reconocimiento requiere.

La inquietud, en su relación con el deseo, tiene aquí la función de dar cuenta de ese movimiento constante que el hombre, en tanto ser finito, realiza a lo largo de la historia con el fin de trascender todo límite: «lo determinado no tiene en tanto tal ninguna otra esencia que esta inquietud absoluta de no ser lo que es» ${ }^{35}$. Y el movimiento de esta inquietud tendrá como resultado, y como principio, la conciencia desgraciada. ¿Puede la conciencia liberarse de esta finitud y de la inquietud que la acompaña?

\section{Ascesis y misticismo ${ }^{36}$}

La dialéctica de la autoconciencia nos presenta así el camino de una autoconciencia singular que no logra escapar a su finitud y deviene conciencia desgraciada. Pero esta conciencia desgraciada tiene todavía un camino que recorrer y que le permitirá alcanzar la universalidad, deshacerse de su determinación propia, de su finitud. Este camino, si bien otorga al trabajo una función importante, quedará signado por el momento de la ascesis ${ }^{37}$ : «La negación debe ir hasta la negación de sí misma - la negación de la negaciónen el ascetismo y la alineación y, así, restaurar lo universal.» ${ }^{38}$ Esta negación de sí es llevada adelante por el asceta ${ }^{39}$ a través de la obediencia y de la renuncia, que trabaja alienando su propia voluntad. Su trabajo no aparece entonces como una realización propia sino como la realización de otro a través del asceta, puesto que éste ha renunciado a sí, a su voluntad y a su goce: «El hombre cree obrar, pero es Dios quien le conduce. [...] El reconocimiento de Dios como el único que obra es la acción esencial del hombre» ${ }^{40}$.

En el caso de Locke, la reflexión sobre la inquietud establecía el problema ético de la libertad. El hombre podía poner en suspenso sus deseos para examinarlos a través del juicio en función de la felicidad futura. Esta posibilidad de hacer intervenir el juicio entre deseos y actos abría el espacio de un trabajo, una práctica, un hábito, a través de los cuales el hombre podía llegar a determinar sus deseos. Ese trabajo constituía una suerte de ascesis, cuya finalidad es el gobierno de los deseos por parte de la conciencia. Aquí, como vemos, las características del asceta son completamente distintas: no se 
trata ya del gobierno de los deseos sino de la renuncia a todo deseo, a toda voluntad propia. El asceta que abre el camino a la universalidad de la razón, tiene como única acción la negación de sí a través de la renuncia y la alienación de su voluntad.

Ahora bien, el pasaje a la universalidad que efectúa el asceta es un momento en un movimiento más amplio y que constituye toda la Fenomenología ${ }^{41}$. En este sentido, la ascesis caracteriza, según Hyppolite, el movimiento completo que describe la totalidad del texto. En el camino de la conciencia hasta el saber absoluto, la existencia completa de dicha conciencia será transformada, $\mathrm{y}$, desde esta perspectiva, se trata de una «ascesis necesaria de la conciencia para llegar al verdadero saber filosófico, ascesis que es toda la Fenomenología» ${ }^{42}$.

La negación de sí que efectúa el asceta, la acción a través de la cual se niega en su finitud para alcanzar la universalidad de la razón, nos enfrenta al problema del misticismo. Este problema atraviesa la obra de Hyppolite por completo y su comentario de la Fenomenología no lo resuelve: ¿se trata allí de un humanismo o un misticismo? La Fenomenología puede dar cuenta del modo en que los hombres hacen la historia, pero puede al mismo tiempo dar cuenta del modo en que lo absoluto se realiza en la historia a través de los hombres: «¿Pueden reconciliarse esos dos aspectos? ¿No plantea el problema fundamental de la teología hegeliana? ¿Cómo se concilian el punto de vista del espíritu finito, el del hombre, y el punto de vista del espíritu infinito, el de Dios?» ${ }^{43}$.

Así, la Fenomenología parece no lograr resolver la unidad entre lo finito y lo infinito. Ahora bien, ¿qué lugar queda para la inquietud en esta filosofía si adoptamos el punto de vista de la infinitud? La dificultad que plantea el dilema entre an- tropología y ontología ¿reduce la inquietud a los hombres individuales mientras que la marcha de Dios transcurre en absoluta serenidad? La respuesta es clara: «Lo infinito no es menos inquieto que lo finito» ${ }^{44}$. Allí radica por otra parte la singularidad de la dialéctica hegeliana: lo infinito, lo absoluto, «esta unidad es "el acto de trascender, no la trascendencia"» ${ }^{45}$. Lo finito no se opone a lo infinito como lo mutable a lo inmutable, a lo eterno inmóvil. Lo infinito no es trascendencia, sino el acto mismo de trascender. La inquietud no sólo está presente tanto en el hombre singular como en el Espíritu universal, sino que además explica el movimiento de ambos. Dicho de otro modo, la inquietud caracteriza propiamente el nivel del pensamiento, de lo espiritual, del concepto: «esta pura inquietud del concepto» ${ }^{46}$, puede decir entonces Hyppolite. Si desde la perspectiva del hombre finito, de la antropología, la inquietud corresponde al deseo de sí, desde el punto de vista de la ontología, la inquietud corresponde al concepto.

Como en Locke, la Unruhe hegeliana se vincula, prima facie, al deseo. Sin embargo, la inquietud sólo caracteriza en Hegel el deseo en la medida en que es deseo de reconocimiento. Esencialmente, la inquietud es del orden de lo espiritual, de modo que no sólo lo finito sino también lo infinito están implicados por aquélla. En el extremo, la inquietud puede caracterizar al concepto mismo. Y, del mismo modo, si ambos autores señalan la necesidad de una ascesis para la consecución de su objetivo, como vimos, las características de esta ascesis son diametralmente diferentes en ambos casos, mientras que Locke nos induce hacia el gobierno de los propios deseos por parte de la conciencia, en Hegel el momento del ascetismo se basa en la renuncia y en la alienación de propio deseo. Por supuesto, la conciencia no es comprendida por Locke 
y por Hegel del mismo modo. Mientras el primero mantiene al respecto una concepción cartesiana, el segundo introduce la intersubjetividad en la formación misma de la conciencia de sí. Esto dará como resultado el problema del misticismo, es decir, la interrogación acerca del estatuto mismo del sujeto. Ya no estamos seguros de que el hombre sea agente, como en Locke, o medio para una subjetividad que lo supera ampliamente: Ser, Dios, Espíritu o Logos: «si podemos todavía hablar de subjetividad, será como de una subjetividad que es el Ser mismo, lo que quiere decir Hegel cuando dice que toda su filosofía se resume en esta frase «lo absoluto es sujeto, y no sustancia» ${ }^{47}$. Pero puesto que lo infinito no es menos inquieto que lo finito, la inquietud misma queda intacta frente a este dilema.

\section{Foucault: «La inquietud del suelo»}

\section{Problematización, pensamiento e inquietud}

El problema que nos deja Hegel en torno al misticismo constituye para nosotros el punto de partida para comprender el modo en que la inquietud funciona en la obra de Foucault. En efecto, también para Foucault la inquietud está ligada al plano del pensamiento ${ }^{48}$, pero el estatuto del mismo es doble. Por un lado, el pensamiento es un êthos, esto es, una forma de ser, de actuar, de hablar y de pensar. Desde este punto de vista, el pensamiento es la racionalidad que organiza en cada caso las prácticas a partir de las cuales tienen lugar los procesos de subjetivación y objetivación de los hombres. Para Foucault, sujetos y objetos no preexisten a las prácticas en las que intervienen, sino que son un efecto de las mismas. Una historia del pensamiento será así una historia de conjuntos prácticos inteligibles de los que se desprenderán modalidades singulares de subjetividad y objetividad. Pero por otro lado, el pensamiento es a su vez «la libertad con respecto a lo que hacemos». Desde este punto de vista, el pensamiento es la posibilidad de tomar distancia con respecto a nuestro êthos y de convertirlo en objeto para su transformación. El primer punto de vista apunta a una ontología (que Foucault llamará «ontología del presente»); el segundo, a una crítica.

De modo que la ambigüedad que hallamos en Hegel tendrá su eco en la obra de Foucault en la noción de pensamiento. Ésta indica a la vez un plano presubjetivo a partir del cual los sujetos serán formados, y un plano subjetivo en el que la reflexividad permite una actividad transformadora y crítica ${ }^{49}$. Ahora bien, la inquietud tiene un lugar en ambos planos, aunque no del mismo modo. En el plano del pensamiento como forma presubjetiva y constituyente, la inquietud, en la medida en que no pertenece ya a ningún sujeto, se encuentra desligada del deseo. La inquietud no pertenece aquí a sujeto alguno, sino más bien al «suelo» sobre el cual el sujeto se levanta: «es a nuestro suelo silencioso e ingenuamente inmóvil al que devolvemos sus rupturas, su inestabilidad, sus fallas; y es él quién se inquieta de nuevo bajo nuestros pasos» ${ }^{50}$.

El establecimiento de un plano de inquietud desligado del deseo no sólo apunta a eliminar la teleología de la historia, sino que tiene además la pretensión de hacer una historia del pensamiento en términos problemáticos, ya que los conjuntos prácticos serán respuestas a esas inquietudes propias de nuestro «suelo». En la «Introducción» de El uso de los placeres, Foucault sostiene que la historia del pensamiento analiza «las problematizaciones a través de la cuales el ser se da como pudiendo y debiendo ser pensado» ${ }^{51}$. El hecho de que el suelo se inquiete, es decir, el hecho de que el ser se nos de bajo una modalidad problemática, no permite suponer en dicho suelo, o en 
el ser, ni un deseo y, por lo tanto, un estadio libre de inquietud, ni una teleología. En El uso de los placeres Foucault pone de manifiesto el hecho de que tanto la cultura contemporánea como la antigua, pasando por el cristianismo, pero también la cultura china, han enfrentado una inquietud similar en torno a la actividad sexual 52

Así, la inquietud no puede tampoco aquí ser considerada como algo anterior o exterior al pensamiento. Aun cuando la inquietud pertenezca al ser, o al suelo, se trata siempre de un plano del pensamiento, por lo que la denominada «ontología del presente», podrá a su vez llamarse «ontología histórica de nosotros mismos». Podemos entonces apreciar la forma que toma ahora el dilema que Hyppolite encontraba en Hegel: no hay inquietud sino en el ámbito del pensamiento y, desde este punto de vista, la inquietud es una inquietud humana, aunque, curiosamente, este pensamiento no sea pensamiento de una conciencia. Hyppolite nos recordaba que si para Hegel todo hombre es conciencia de sí, la fórmula no puede invertirse para decir que toda conciencia de sí sea un hombre. Foucault opera una inversión de otro tipo: si bien toda conciencia de sí se da en un hombre, no todo lo humano es conciencia de sí; el pensamiento como suelo, como elemento constituyente de lo humano, no es una conciencia de sí.

\section{Inquiétude y deseo}

Ahora bien, si la inquietud se desliga del deseo en el plano del pensamiento que caracteriza nuestro êthos, en el plano subjetivo del pensamiento, inquietud y deseo se reencuentran en un espacio abierto de relaciones de poder. En El orden del discurso, conferencia inaugural en el Collège de France, Foucault comienza expresando un deseo: «Más que tomar la palabra, habría preferido verme envuelto por ella y transportado más allá de todo comienzo posible» ${ }^{53}$. Y a este deseo, la institución da una respuesta: «No tienes que temer el comenzar, todos estamos aquí para mostrarte que el discurso está en el orden de las leyes»» ${ }^{54}$. Lo que en el plano del pensamiento en tanto êthos se presentaba como inquietud fundamental dándose a los hombres como problema, en el plano subjetivo del pensamiento da lugar a deseos y respuestas. El deseo de Foucault y la respuesta de la institución, son así

«réplicas opuestas a una misma inquietud: inquietud con respecto a lo que es el discurso en su realidad material de cosa pronunciada o escrita; inquietud con respecto a esta existencia transitoria destinada a borrarse sin duda, pero según una duración que no nos pertenece; inquietud de sentir bajo esta actividad, sin embargo cotidiana y gris, poderes y peligros que imaginamos con dificultad; inquietud de sospechar la existencia de luchas, victorias, heridas, dominaciones, servidumbres, a través de tantas palabras cuyo uso, después de tanto tiempo, ha reducido las asperezas» ${ }^{55}$.

Los deseos y respuestas ante las inquietudes de nuestro suelo, se encuentran enmarcadas por los modos de problematización que encontramos en cada época, y en cada lugar ${ }^{56}$. Ahora bien, si con Locke vimos que la ausencia de un bien no necesariamente implica la inquietud que nos llevará a perseguirlo, mientras que la presencia de un mal nos imponía el deseo de librarnos de él, Foucault seguirá este principio para intervenir en el ámbito de las relaciones de poder ${ }^{57}$. En la frase de Las palabras y las cosas citada más arriba, vimos que se trataba de devolver al suelo su inquietud. La noción de lo intolerable se encargará de esta tarea a la vez histórica y política.

Lo intolerable juega un papel importante en la obra de Foucault. Sin ser un concepto stricto sensu, encontramos elementos que pueden servir a su conceptualización. En primer lugar, lo intolerable 
reclama un trabajo a la vez histórico y político. Desde el punto de vista histórico, supondríamos que lo intolerable no hace más que dar cuenta de la «sensibilidad» de una sociedad en un período determinado; se constituye así como una suerte de «índice» que podría ayudar a medir un progreso moral en la humanidad. Desde el punto de vista político, supondríamos que aquello que nuestra sensibilidad establece como intolerable constituye una suerte de límite al poder: por definición, lo intolerable no es tolerado. Para Foucault, en cambio, lo intolerable implica a la política en la medida en que se trata también de un asunto «de resistencia, de capacidad de rechazo y de voluntad de combate» ${ }^{58}$. Así, la perspectiva histórica debe fundirse con el análisis político para introducir el espacio en el tiempo, de modo que a la sucesión diacrónica de las sensibilidades se agregue el análisis sincrónico de los conflictos y prácticas de los que lo intolerable es resultado. En el plano histórico, la introducción del espacio político no nos dará como resultado una «sensibilidad» epocal, sino el registro y la memoria de las luchas frente a las cuales algunas cosas se vuelven intolerables y otras tolerables. En el plano crítico del pensamiento, historia y política se funden en una estrategia que tiene por objetivo modificar las relaciones de poder presentes ${ }^{59}$ devolviendo la inquietud allí donde «el hábito y la falta de análisis» habían vuelto invisible lo problemático, y aumentar la intolerancia frente a los efectos de poder ejercidas a través de prácticas cotidianas.

\section{Inquietud y ascesis}

Hemos visto que lo que se presenta como inquietud bajo una determinada modalidad problemática en cada momento y lugar en el plano asubjetivo y ontológico del pensamiento, da lugar a deseos y respuestas en el plano subjetivo y crítico.
Sin embargo, no por ello debemos suponer que las respuestas sean una solución a lo problemático. Foucault rechaza la idea de una sociedad sin inquietudes ni conflictos ${ }^{60}$, así como la idea de una finalidad en la historia por la cual habría que tolerar y atravesar los mecanismos de poder actuales. Si, por un lado, nunca se sale de la inquietud ni de la problematicidad, por otro, no hay legitimidad intrínseca para ninguna relación de poder ${ }^{61}$. Dicho de otro modo, la inquietud es inevitable, pero ningún conjunto práctico establecido como respuesta es necesario. Por esto, el principio de una inquietud permanente propia de lo humano no tiene por qué implicar resignación o aceptación del estado de cosas. Tomar la permanencia de la inquietud como una suerte de factum, o como diagnóstico, no debe hacernos olvidar el carácter frágil y polémico del presente: «Ocurre que la gente toma este esfuerzo de reproblematización como un "antirreformismo" que reposaría sobre un pesimismo del tipo "nada cambiará". Es todo lo contrario» ${ }^{62}$.

Frente a la inquietud constante Foucault no da lugar a una «liberación» que nos garantizaría la serenidad, sino más bien a una ascesis ${ }^{63}$ y a lo que Foucault llamará prácticas de libertad. En efecto, sus últimos trabajos están enmarcados por las prácticas de sí, es decir, por las prácticas a través de la cuales los individuos se constituyen a sí mismos como sujetos. Las formas a través de las cuales los hombres han buscado transformarse a sí mismos son múltiples. Sin embargo, a partir de sus análisis de la antigüedad, Foucault toma dos modelos: Eros y Askesis ${ }^{64}$. En este par, que podemos traducir por «amor» y «trabajo», se juega la estrategia para enfrentar la inquietud del pensamiento. El Eros, por su parte, pone en juego una conversión (conversion) de la que la revolución moderna será heredera. Ya Hegel había considerado al amor 
como una posibilidad de enfrentar la escisión de la conciencia desgraciada, pero finalmente optó, como sabemos, por el trabajo de lo negativo a través de la renuncia del asceta ${ }^{65}$. Foucault, cuyas dudas acerca de la revolución no son menores a las de Hegel, también elegirá el trabajo como práctica ascética de transformación de sí a través de prácticas de libertad ${ }^{66}$. Esta ascesis no tomará ni la forma de una renuncia de sí, como en Hegel, ni de un trabajo con la pretensión de alcanzar la maîtrise de soi, como en Locke, o como encontramos en las filosofías de la antigüedad sobre las que Foucault trabajará en el final de su vida. De hecho, no es posible dar una definición de esta ascesis que pueda servir como criterio normativo o programático. Los elementos con los que contamos para caracterizar la forma que la ascesis cobra en la obra del francés, dan cuenta de la imposibilidad para determinarla de modo definitivo: son las nociones de ensayo y de experimentación.

Tanto en el caso del ensayo como en el de la experimentación, la filosofía se encontrará en el centro del planteo. El primer caso lo encontramos en la «Introducción» de El uso de los placeres, donde la filosofía es presentada como una ascesis cuyo cuerpo sería, precisamente, el ensayo: «El "ensayo" - que hay que entender como prueba modificadora de sí mismo en el juego de la verdad [...] - es el cuerpo vivo de la filosofía, al menos si ésta es lo que fue antes, es decir una ascesis, un ejercicio de sí, en el pensamiento» ${ }^{67}$. El segundo caso, lo encontramos en el êthos filosófico que caracteriza la actitud de la modernidad en el ya célebre opúsculo «¿Qué es la Ilustración?»: «Me parece que esta actitud histórico-crítica debe ser también una actitud experimental» 68 . Esta actitud deberá apoyarse más una voluntad de experimentación y ensayo que en principios a seguir. En efecto, estos principios sólo podrían surgir de nuestro modo actual de ser, de modo que resultarían guías dudosos para el intento de «pensar de otro modo» ${ }^{69}$. La ascesis que nos sugiere Foucault apunta al trabajo de una actitud:

«[...] exigente, prudente, "experimental"; en necesario a cada instante, paso a paso, confrontar lo que pensamos y lo que decimos a lo que hacemos y a lo que somos [...] Si yo mantuve esta "práctica", no es para "aplicar" ideas, sino para ponerlas a prueba y modificarlas» ${ }^{70}$.

Así, esta práctica ascética es situada en el eje mismo de un trabajo filosófico cuya finalidad es adquirir un nuevo modo de pensamiento para responder a las inquietudes del suelo sin someterse a prácticas cuyos efectos de poder se perciben como necesarios. El trabajo histórico-político permitirá así poner en evidencia la no-necesidad de estos efectos y hará posible percibirlos como intolerables.

\section{Conclusión}

Cuando nos referimos a la inquietud, si bien damos cuenta a una falta de quietud, comodidad o tranquilidad por cuestiones que pueden ir desde cierta preocupación hasta la desgracia de la conciencia, lo que se impone como denominador común es el hecho de que la situamos en el plano del pensamiento. La inquietud no pertenece ni a las cosas ni a la naturaleza, sino al ámbito de lo humano.

Es innegable que, históricamente, esta noción ha estado ligada al deseo. Para Locke, la uneasiness y el deseo se implican mutuamente. Para Hegel la conciencia de sí, la autoconciencia, es a la vez deseo de sí e inquietud. Pero la forma en que Foucault concibe la inquietud del «suelo» nos muestra que el vínculo con el deseo no es necesario. Esto podría verse ya en el problema del misticismo que Hyppolite reconoce en la filosofía hege- 
liana, si no fuera porque lo absoluto, para el alemán, es concebido como un sujeto. Así, incluso el plano lógico del concepto puede ser caracterizado de inquieto. Pero con Foucault, hemos visto que el plano del pensamiento, que corresponde a las prácticas intersubjetivas de las cuales los sujetos individuales son efecto, no tiene por qué ser concebido como una conciencia de sí cuya voluntad persiga un fin determinado. Es cierto que aún en Foucault la inquietud puede habilitar un deseo por parte del individuo, pero ese deseo es más bien una respuesta, nunca perfecta, frente a lo que inquieta y se presenta como problemático. De suerte que si para Locke o Hegel la inquietud es la fuente humana de un movimiento cuyo fin es terminar con la inquietud misma, en Foucault es más una herramienta para el diagnóstico del presente y la transformación de sí.
En este sentido, hemos visto que hay otro elemento que no deja de presentarse en estos autores y que puede considerarse como correlativo a la inquietud del pensamiento: la ascesis. Las formas que esta toma en cada autor se deben, en gran medida, a la concepción de la subjetividad y del estatuto del pensamiento: dominio de sí en la conciencia cartesiana de Locke, renuncia al yo singular que habilita el pensamiento de lo universal en Hegel; y trabajo experimental, que busca escapar tanto a un sujeto soberano como a una resignada renuncia al sujeto, que nos permita lidiar con una inquietud permanente. Este vínculo con la ascesis no puede extrañar. A diferencia de la noción de angustia, ligada al problema del sentido, o de la de contradicción ligada a la lógica del ser, hemos visto que, en cada uno de los autores tratados, la inquietud se sitúa frente al problema del poder y de la libertad.

\section{NOTAS}

${ }^{1}$ Los trabajos de Wittgenstein y de Foucault en torno a la noción de juego constituyen un caso de este tipo.

2 D. Fassin, Quand les corps se souviennent, Expériences et politiques du sida en Afrique du Sud, Paris, Éditions La Découverte, 2006.

3 W. H. Auden, The Age of Anxiety: A Baroque Eclogue, New York, 1947.

${ }^{4}$ E. Castelli Gattinara, Les inquiétudes de la raison. Epistémologie et histoire en France dans l'entre-deux-guerres, Paris, Vrin EHESS, 1998.

5 J.-L. Nancy, Hegel: L'inquiétude du négatif, XXX, Hachette, 1997; y M. Potte-Bonneville, Michel Foucault: L'inquiétude de l'histoire, Paris, PUF, 2004.

6 Esto tiene lugar en el Libro II (Of Ideas), cuyo capítulo más extenso, el XXI, lleva como título «Del poder» (Of Power), aunque su tema sea de hecho la libertad. La noción de inquietud (uneasiness) es presentada en el capítulo XX, «De los modos de placer y dolor» (Of Modes of Pleasure and Pain). Las traducciones son nuestras, a no ser que se indique lo contrario.

7 Ibíd., $§ 5$.

8 Ibíd., § 8 .

9 Ibíd., § 9.
10 Ibíd., § 8 .

11 «For how can we think any one freer, than to have the power to do what he will?», ibíd., § 21 .

12 Cf. Ibíd., § 29.

13 Ídem.

14 Cf. John Locke, Ensayo sobre el entendimiento humano, traducción de Edmundo O'Gorman, México, Fondo de Cultura Económica, 1956, pp. 232-268.

15 Diego Vernazza ha mostrado que Leibniz introduce esta noción al pensamiento alemán a partir de la traducción francesa de la uneasiness lockeana como inquiétude.

16 John Locke, An essay concerning human understanding, Book II, Chapter XXI, § 31 .

17 En efecto, el $\S 32$ lleva como título Desire is Uneasiness.

18 «Veo lo mejor, lo apruebo y sigo lo peor». Locke cita aquí a Ovidio (Metamorph., lib. VII, v. 20-21), ibíd., § 35 .

19 Ibíd., § 56.

20 Ibíd., § 41.

21 Ibíd., § 42.

22 Ibíd., § 48.

23 Ibíd., § 51.

24 Cf. ibíd., § 61 .

25 Ibíd., § 69 . 
26 Utilizamos la noción de ascesis en el sentido amplio que le dará Foucault, como «ejercicio de sí sobre sí por el cual se trata de elaborarse, de transformarse y de acceder a un cierto modo de ser», Cf. «L'éthique du souci de soi comme pratique de la liberté», en Dits et Ecrits vol. IV, Paris, Gallimard, 2001, p. 709 . En adelante se citará $D E$ y el número de volumen en números romanos.

27 La traducción de Hyppolite de la Fenomenología del espíritu constituyó sin dudas un acontecimiento singular en Francia; al respecto, Althusser declara: «Nous avons une très grande dette à l'égard de $M$. Hyppolite [...] Nous lui devons de connaître Hegel», J. Hyppolite (comp.), Hegel et la pensée moderne, $\mathrm{Pa}-$ ris, PUF, 1970 , p. 85. Para más detalles sobre la recepción de Hegel en Francia, cf. Bernard Bourgeois, «Hegel en France», en Olivier Tinland (dir.), Lectures de Hegel, Paris Cedex, 2005, pp. 36-59.

28 Al respecto, Foucault dirá más tarde: «Escuchábamos algo de la voz de Hegel, y quizá, más aún, la voz de la filosofía misma». M. Foucault, $D E I$, p. 807.

${ }^{29} D E I$, p. 813. Para más detalles sobre la relación de Foucault con Hyppolite puede verse Eribon D., Michel Foucault, Paris, Flammarion, 2008, especialmente el capítulo «La voix de Hegel».

${ }^{30}$ Cf., M. Foucault, L'ordre du discours, Paris, Flammarion, 1971, pp. 79-82.

31 J. Hyppolite, Génesis y estructura de la Fenomenología del espiritu, Traducción de Francisco Fernández Buey, Barcelona, Península, 1998, p. 176.

32 Ibíd., p. 145.

33 Ibíd., p. 151. La cursiva es nuestra.

34 Ibíd., pp. 136-137. El «comentador reciente» al que se refiere Hyppolite es $\mathrm{H}$. Marcuse y su tesis $\mathrm{He}$ gels Ontologie und die Grundleung einer Theorie der Geschichlichkeit (1932).

35 «Vie et prise de conscience de la vie dans la philosophie hégélienne d'Iéna», en J. Hyppolite, Études Sur Marx et Hegel, Paris, Librairie Marcel Rivière et Cie., 1955, p. 18.

36 Tomamos la noción de misticismo en el sentido que le otorga el helenista Pierre Hadot en función de Lalande: «Creencia en la posibilidad de una unión íntima y directa del espíritu humano con el principio fundamental del ser, unión que constituye a la vez un modo de existencia y un modo de conocimiento extraños y superiores a la existencia y al conocimiento normales». Cf. P. Hadot, «Les niveaux de conscience dans les états mystiques selon Plotin», en Journal de psychologie n. ${ }^{\circ} 2-3,1980$, pp. 243-244.

37 Por cuestiones de espacio no corresponde aquí desarrollar todos los momentos por los que pasa la autoconciencia del esclavo hasta devenir razón. Nos centramos simplemente en lo principal del último momento de la dialéctica, que dará lugar a la razón universal y de este modo escapará a la finitud de la singularidad.

38 J. Hyppolite, Génesis y estructura de la Fenomenología del espiritu, p. 189. Sin duda habría que analizar aquí la forma con que el trabajo aparece en la Fenomenología. Es sabido que para el marxismo éste será uno de los pilares de la inversión del esquema hegeliano. Sin embargo, nos limitamos aquí a señalar dos cuestiones: 1) el trabajo al que se refiere Hegel no es «formador» para la conciencia sino en la medida en que surge de la angustia y alcanza la realización de un producto capaz de permanecer en términos espirituales (es decir que está más cerca del arte que de la producción industrial por parte de un proletariado); y 2) no se puede olvidar el hecho de que al momento del trabajo y transformación del mundo por parte del esclavo debe seguir el acto de renuncia por el cual éste reconoce que sólo Dios es quien obra.

39 «Si la verdad de la conciencia esclava era la conciencia estoica, la verdad de la conciencia desgraciada será la conciencia del asceta, del santo que se propone anular su propia singularidad para devenir así una autoconciencia más profunda». Ibíd., p. 192.

40 Ibíd., pp. 190-191

${ }^{41}$ Hyppolite resume, desde este punto de vista, la Fenomenología como el camino transformador «del yo al nosotros», ibíd., p. 345.

42 Ibíd., p. 15. (La cursiva es nuestra).

43 Ibid., p. 490. Hyppolite mantiene la tensión que encuentra en Hegel sin dar una respuesta definitiva: «Podemos prolongar la filosofía hegeliana en dos direcciones diferentes: una filosofía que termina en un humanismo, en el sentido de Feuerbach o de Marx, o una filosofía del saber absoluto: pero en este último caso, ¿qué significa todavía la historia de la humanidad?»; «Ruse de la raison et histoire chez Hegel», en J. Hyppolite, Figures de la pensée philosophique, tome I, Paris, PUF, 1971, p. 150.

44 J. Hyppolite, Génesis y estructura de la Fenomenología del espíritu, p. 138.

45 «Vie et prise de conscience de la vie dans la philosophie hégélienne d'Iéna», en J. Hyppolite, Etudes Sur Marx et Hegel, Paris, Librairie Marcel Rivière et Cie., 1955, p. 18.

46 «Situation de l'homme dans la phénoménologie hégélienne», en J. Hyppolite, Études sur Marx et Hegel, Paris, Librairie Marcel Rivière et Cie., 1955, p. 178 .

47 «Essai sur la "Logique" de Hegel», en J. Hyppolite, Figures de la pensée philosophique tome I, Paris, PUF, 1971, p. 163.

48 Es mérito de M. Potte-Bonneville el haber puesto de relieve la importancia de la inquietud en la obra de Foucault; y en ese sentido, este trabajo explora una línea abierta por él, aunque la perspectiva no sea idéntica. Cf. M. Potte-Bonneville, Michel Foucault, L'inquiétude de l'histoire, Paris, PUF, 2004. Potte-Bonneville utiliza esta noción poniendo el acento en un aspecto biográfico-subjetivo del propio Foucault para ubicarlo en el panorama filosófico del siglo xx y, en particular, para marcar sus diferencias con las obras de Heidegger y de Sartre, para quienes la angustia pasa a ocupar el lugar privilegiado. 
49 Puesto que nuestro interés se centra en la noción de inquietud, no evaluaremos aquí la consistencia del planteo de Foucault en este punto. Para esto cf. B. Han, L'ontologie manquée de Michel Foucault, Grenoble, Editions Jerome Millon, 1998.

50 M. Foucault, Les mots et les choses, Paris, Gallimard, 1966, p. 16.

51 M. Foucault, Histoire de la sexualité II: L'usage des plaisirs, Paris, Gallimard, 1984, p. 17. En adelante se citará UP.

52 Cf. ibíd., pp. 153-156.

53 M. Foucault, L'ordre du discours, Paris, Gallimard, 1971, p. 7.

54 Ibíd., p. 9.

55 Ibíd., pp. 9-10.

56 Señalamos más arriba la inquietud que diversas culturas encontraron en la actividad sexual. Sin embargo, sus respuestas han sido distintas en función del modo en que esta inquietud podía y debía pensarse en cada caso. Mientras que los griegos habrían elaborado una estética de la existencia, el cristianismo habría respondido a través de una codificación jurídico-moral alrededor de la noción de carne (chair), la modernidad lo habría hecho a través del dispositivo de sexualidad, y la cultura china habría elaborado como respuesta todo un arte erótica (ars erotica). Cf. UP, p. 156.

57 Desde este punto de vista, cabe recordar que la obra de Foucault tiene intenciones políticas expresas. Baste recordar la figura del intelectual específico y la imagen de la caja de herramientas.

$58 D E I V$, p. 35.

59 Ante una pregunta por la investigación que Foucault lleva adelante con el Grupo de Información sobre las Prisiones (GIP), el filósofo respondía: «Nuestra investigación no se hace para acumular conocimientos, sino para aumentar nuestra intolerancia y hacer de ella una intolerancia activa. Devengamos intolerantes en relación a las prisiones, a la justicia, al sistema hospitalario, de la práctica psiquiátrica, etc.» DE II, p. 176.

60 «Una sociedad "sin relaciones de poder" no puede ser sino una abstracción». $D E I V$, p. 239.

61 Ídem.

62 «À propos de la généalogie de l'éthique: un aperçu du travail en cours», en Dits et Ecrits II, p. 1431 .

63 «Es lo que se podría llamar una práctica ascética, dando al ascetismo un sentido muy general, es decir no el sentido de una moral de la renuncia, sino el de un ejercicio de sí por sí por el cual se intenta elaborarse, transformarse y acceder a un cierto modo de ser». "L'éthique du souci de soi comme pratique de la liberté», en $D E I V$, p. 709.

64 Cf. M. Foucault, Herméneutique du sujet, Paris, Seuil/Gallimard, 2001, p. 17.

65 J. Hyppolite, Génesis y estructura de la Fenomenología del espíritu, p. 149.

66 Cf. ibíd., pp. 709-712.

${ }^{67}$ UP, p. 15. Esta concepción de la filosofía como práctica ascética es tomada del helenista Pierre Hadot. Cf. de este autor Exercices spirituels et philosophie antique, Paris, Institut d'Études Augustiniennes, 1993, y Qu'est que la philosophie antique?, Paris, Gallimard, 1995.

Cf. M. Foucault, Herméneutique du sujet, Paris, Seuil/Gallimard, 2001, p. 17.

68 DE IV, p. 574.

69 «Pero ¿qué es la filosofía hoy — quiero decir la actividad filosófica - si no es el trabajo crítico del pensamiento sobre sí mismo? ¿Y si no consiste, en lugar de legitimar lo que ya sabemos, en emprender la tarea de saber cómo y hasta qué punto sería posible pensar de otro modo?».

70 «Politique et éthique: une interview», en $D E I V$, p. 535 . 